\title{
INFLUÊNCIA DO TEMPO DE USO SOBRE AS CARACTERÍSTICAS HIDRÁULICAS DO MICROASPERSOR DO GRUPO MODULAR
}

\author{
DELVIO SANDRI ${ }^{1}$, MÁRCIO MESQUITA ${ }^{2}$, KADY S. E. BESSA ${ }^{3}$, \\ LÁZARO C. R. A. PRADO ${ }^{4}$
}

\begin{abstract}
RESUMO: O desenvolvimento de novos equipamentos de irrigação e o aprimoramento dos já existentes, como dos microaspersores, devem considerar a avaliação das características hidráulicas, disposição adequada dos mesmos em condições de campo e interferência devido ao tempo de uso, garantindo índices de desempenho satisfatório. Nesse contexto, este trabalho teve o objetivo de determinar a equação vazão x pressão, o coeficiente de variação de fabricação - CVf e de vazão $\mathrm{CVq}$, a uniformidade de distribuição de água para diferentes graus de sobreposição do microaspersor do grupo modular de bocal laranja, novo e usado, sob funcionamento na posição invertida. $\mathrm{O}$ aumento do espaçamento entre microaspersores e entre laterais reduziu os índices de uniformidade em todas as pressões estudadas, tanto do microaspersor novo como do usado. A maior precipitação do jato de água ocorreu na distância de 0,0 a $1,0 \mathrm{~m}$ da haste de sustentação dos microaspersores novo e usado. $\mathrm{O}$ alcance do jato de água do microaspersor usado foi menor em relação ao bocal novo.
\end{abstract}

PALAVRAS-CHAVE: produção de mudas, irrigação localizada, ambiente protegido.

\section{INFLUENCE OF TIME OF USE ON HYDRAULIC CHARACTERISTICS OF MICROSPRINKLER OF MODULATE GROUP}

\begin{abstract}
The development of new irrigation equipments and the improvement of the already existent, as the microsprinkler, demand to evaluate the hydraulic characteristics as well as the appropriate installation in the field conditions to obtain higher indexes of the water distribution uniformity. This work had the objective to obtain the discharge $\mathrm{x}$ pressure equation, the manufacturing variation coefficient - CVf and the discharge variation coefficient - CVq, water distribution uniformity for different overlap degrees of the microsprinkler of the modulate group, orange nozzle, new and used, installed in the upside-down at $1.20 \mathrm{~m}$ above laboratory ground. The increase of the spacing among microsprinkler and lateral reduced the uniformity indexes for all of the pressures for the new and the used microsprinkler. The high jet concentration occurred in the range from 0.0 to $1.0 \mathrm{~m}$ of the support rod of the new and used microsprinkler. The wetted area by the used microsprinkler was smaller than the new one.
\end{abstract}

KEYWORDS: seedling, trickle irrigation, greenhouse.

\section{INTRODUÇÃO}

Para atender às demandas de uma agricultura moderna, novos equipamentos são fabricados com os mais variados tipos de máquinas e materiais, implicando a necessidade da determinação de suas características estruturais e operacionais (SILVA \& SILVA, 2003), como em microaspersores, que em estufa normalmente operam em posição invertida. Na maioria das vezes, são cultivadas plantas de alto valor comercial nesses ambientes e por estarem em uma condição sensível à falta de água e às condições ambiente, exige sobreposição adequada do jato de água, bem como elevados

\footnotetext{
${ }^{1}$ Prof. DE V, Faculdade de Engenharia Agrícola, UnUCET, UEG, BR 153, Caixa Postal 459, Anápolis - GO, sandri@ ueg.br.

${ }^{2}$ Doutorando em Engenharia Agrícola, FEAGRI, UNICAMP, Campinas - SP, marcio.mesquita@ feagri.unicamp.br.

${ }^{3}$ Eng ${ }^{\mathrm{O}}$ Agrícola, UnUCET, UEG, Anápolis - GO, kseb40@ hotmail.com.

${ }^{4}$ Mestrando, IESA-Geografia, UFG, Goiânia - GO, lazaroagricola@gmail.com.

Recebido pelo Conselho Editorial em: 1ํ--2008

Aprovado pelo Conselho Editorial em: 2-10-2010
} 
coeficientes de uniformidade de distribuição e lâmina aplicada apropriada, grandemente dependente da disposição e desempenho dos microaspersores, tanto novos quanto usados.

Com o aumento de área irrigada, houve uma expansão do mercado de dispositivos fabricados, a fim de atenderem a esta demanda, surgindo novas empresas e produtos, exigindo estudos sobre o funcionamento dos mesmos, bem como estabelecer novos parâmetros para testes mais eficazes e de menor custo (MAZZER et al., 2003).

Para SIQUEIRA \& BARROS JÚNIOR (2003), a principal finalidade de ensaios de equipamentos utilizados em sistemas de irrigação localizada consiste na determinação e avaliação de suas características hidráulicas. São muitos os fatores que influenciam ou afetam a eficiência de aplicação d'água, destacando-se: variação da vazão devido ao processo de fabricação; expoente da pressão na equação de vazão do emissor e estabilidade da vazão em função da pressão; variação da pressão de funcionamento; perda de carga em razão da inserção do emissor na linha lateral e suscetibilidade a entupimento por sedimentação ou precipitação de sais.

A falta de informações sobre os emissores e a dificuldade de escolha da motobomba mais adequada podem acarretar consequências sérias, como o consumo excessivo de energia, sobrecarga no motor, entupimentos, desuniformidade de emissão e abandono do sistema, retornando a métodos de aplicação menos eficientes (SOUZA et al., 2005). A introdução de microaspersores para irrigação de cultivos protegidos afetou profundamente este conceito, que serviu de base para o desenvolvimento desta tecnologia. Em se tratando de cultivos de alto valor comercial que requerem um nível muito preciso de controle, exige-se que também as aplicações de água sejam mais uniformes possíveis (SAPIR \& SNEH, 2002).

A uniformidade da irrigação tem efeito no rendimento das culturas e é considerada um dos fatores mais importantes no dimensionamento e na operação de sistemas de irrigação. A uniformidade de distribuição de água apresenta, em qualquer sistema de irrigação, importantes conseqüências na economia do projeto. Reduzidos valores de uniformidade determinam em geral, maiores consumo de água e energia, maior perda de nutrientes e, ao mesmo tempo, podendo propiciar déficit hídrico ás plantas (SCALOPPI \& DIAS, 1996).

Segundo ASSAD (1994) existe uma grande preocupação quanto ao uso da água para irrigação, e geralmente sua demanda aumenta nos períodos mais secos. Considerando a importância do Cerrado, e a necessidade de que os recursos hídricos sejam utilizados de forma racional, é essencial utilizar sistemas de irrigação, que sejam eficientes na aplicação de água, já que a irrigação exige uma grande demanda de água. Para obtenção de resultados satisfatórios na irrigação localizada são necessárias informações detalhadas sobre a uniformidade de distribuição de água e raio de alcance do jato de água dos emissores.

A exemplo de outras regiões do Brasil, o Cerrado, grande produtor agrícola, com grande utilização dos recursos hídricos na irrigação, por ocasião de estiagens sazonais, tem resultado em conflitos de uso para atender à irrigação, que é o maior consumidor, com cerca de $46 \%$ do volume captado, com a indústria e, principalmente, com o abastecimento público, ocorrendo tanto em importantes polos urbanos, como no Distrito Federal, Goiânia, Anápolis e Palmas e também em cidades de menor porte (BRASIL, 2007).

Dentre as novas tecnologias voltadas para agricultura irrigada, a prática da agricultura em ambientes protegidos tem-se destacado, tendo como principais vantagens a garantia da colheita, produtos de melhor qualidade, uso racional de pequenas áreas, dentre outras (QUEIROZ, 2004).

As exigências quanto ao uso dos recursos hídricos justificam qualquer ação de pesquisa que envolva melhoria no manejo da irrigação. Para SANTIAGO et al. (2004), a expansão da agricultura irrigada tem-se tornado preocupante, devido ao elevado consumo e às restrições de disponibilidade de água, bem como ao risco de degradação do sistema solo-água-planta. 
Este trabalho teve como objetivo avaliar as características hidráulicas e simulação da uniformidade de distribuição de água do microaspersor do grupo modular, sob diferentes condições de uso.

\section{MATERIAL E MÉTODOS}

O experimento foi realizado no Laboratório de Hidráulica da Unidade Universitária de Ciências Exatas e Tecnológicas (UnUCET) da Universidade Estadual de Goiás (UEG), Anápolis-GO.

Avaliou-se o microaspersor do grupo modular, bocal laranja, com dispositivo giratório de longo alcance, novo e usado, marca NaanDan, seguindo a norma da ABNT (1986). O diâmetro do bocal é de $1,5 \mathrm{~mm}$, promovendo um diâmetro molhado de $8,5 \mathrm{~m}$, quando instalado na posição invertida e a $1,8 \mathrm{~m}$ do solo e com vazão de $120 \mathrm{~L} \mathrm{~h}^{-1}$ sob pressão de $200 \mathrm{kPa}$, segundo catálogo do fabricante. Os microaspersores ensaiados possuem 7 anos de uso em ambiente protegido, funcionando na posição invertida, objetivando a produção de mudas de hortaliças. O microaspersor é classificado como não autocompensante e não setorial.

Avaliou-se um emissor por vez, instalado em uma tubulação de 3/4" e a $1,20 \mathrm{~m}$ do piso do laboratório, sendo a pressão regulada com um registro de agulha de 3/4" e medida com um manômetro de Bourdon, com precisão de $2 \mathrm{kPa}$. Utilizou-se, no ensaio, um reservatório com capacidade de $0,50 \mathrm{~m}^{3}$, conectado por tubulação a uma motobomba centrífuga, marca Thebe, modelo THS-18, $3 \mathrm{CV}$, trifásica, $60 \mathrm{~Hz}$ e $3.500 \mathrm{rpm}$.

Para determinar o coeficiente de variação de fabricação - CVf e de vazão - CVq, avaliaram-se individualmente 20 microaspersores, com um tempo de coleta de 2 minutos, com auxílio de um cronômetro digital, com três repetições, obtendo-se a média geral de todos os microaspersores e a média individual de cada um deles para cada pressão de serviço.

Determinou-se a equação que relaciona a vazão x pressão dos microasepersores, com os dados de 10 microaspersores com vazão mais próximos da média geral, na pressão de $200 \mathrm{kPa}$. As pressões usadas foram as de 150;200; 250; 300 e $350 \mathrm{kPa}$.

O coeficiente de variação de fabricação - CVf (peças novas) e de vazão - CVq (peças usadas) foi obtido pela Equação 1, descrita por KELLER \& BLEISNER (1990).

$$
\mathrm{CVf} \text { ou } \mathrm{CVq}=\frac{\mathrm{S}}{-} 100
$$

em que,

CVf - coeficiente de variação de fabricação, \%;

$\mathrm{CVq}$ - coeficiente de variação de vazão, \%;

$\mathrm{S}$ - desvio-padrão da vazão média dos emissores, $\mathrm{L} \mathrm{h}^{-1}$, e

$\bar{q}$ - vazão média a uma pressão e temperatura ambiente, $\mathrm{L} \mathrm{h}^{-1}$.

A classificação do coeficiente de fabricação de emissores de saída pontual, segundo a ASAE (1994) é: <0,05, excelente; entre 0,05 e 0,07, médio; entre 0,07 e 0,11, marginal; entre 0,11 e 0,15, deficiente e $>0,15$, inaceitável. Pela ABNT (1986) é: <10\%, boa; entre 10 e 20\%, média; entre 20 e $30 \%$, marginal e $>30 \%$, inaceitável, e pela ISO 9260/1 (1991) é: $<0,05$, categoria A e entre 0,05 e 0,1 , categoria B.

A vazão precipitada em cada coletor, durante uma hora de ensaio, foi medida pelo método gravimétrico, com uma balança eletrônica digital, marca METTLER, modelo PE3600, com precisão de 0,01 g. Determinou-se a vazão de 20 emissores, conforme sugerido pela Norma 12: 02.08 - 021 da ABNT (1986). Em seguida, foram selecionados os três microaspersores com vazão mais próxima da vazão média para a pressão de $200 \mathrm{kPa}$, para avaliação da uniformidade de distribuição, 
correspondendo às três repetições. A vazão em cada ponto de coleta dentro do raio de alcance do jato do microaspersor foi obtida pela média da vazão dos três microaspersores para cada pressão de serviço.

A lâmina de irrigação foi obtida utilizado um kit de ensaio de precipitação, com área de coleta de $0,00504 \mathrm{~m}^{2}$ e altura de $0,102 \mathrm{~m}$. Os coletores foram instalados sobre o piso plano do laboratório, dispostos em $0,5 \mathrm{~m} \times 0,5 \mathrm{~m}$ (Figura 1), formando uma malha quadrada, conforme sugere a Norma 12: 02.08 - 021 da ABNT (1986), de modo a cobrir toda a área molhada pelo emissor.

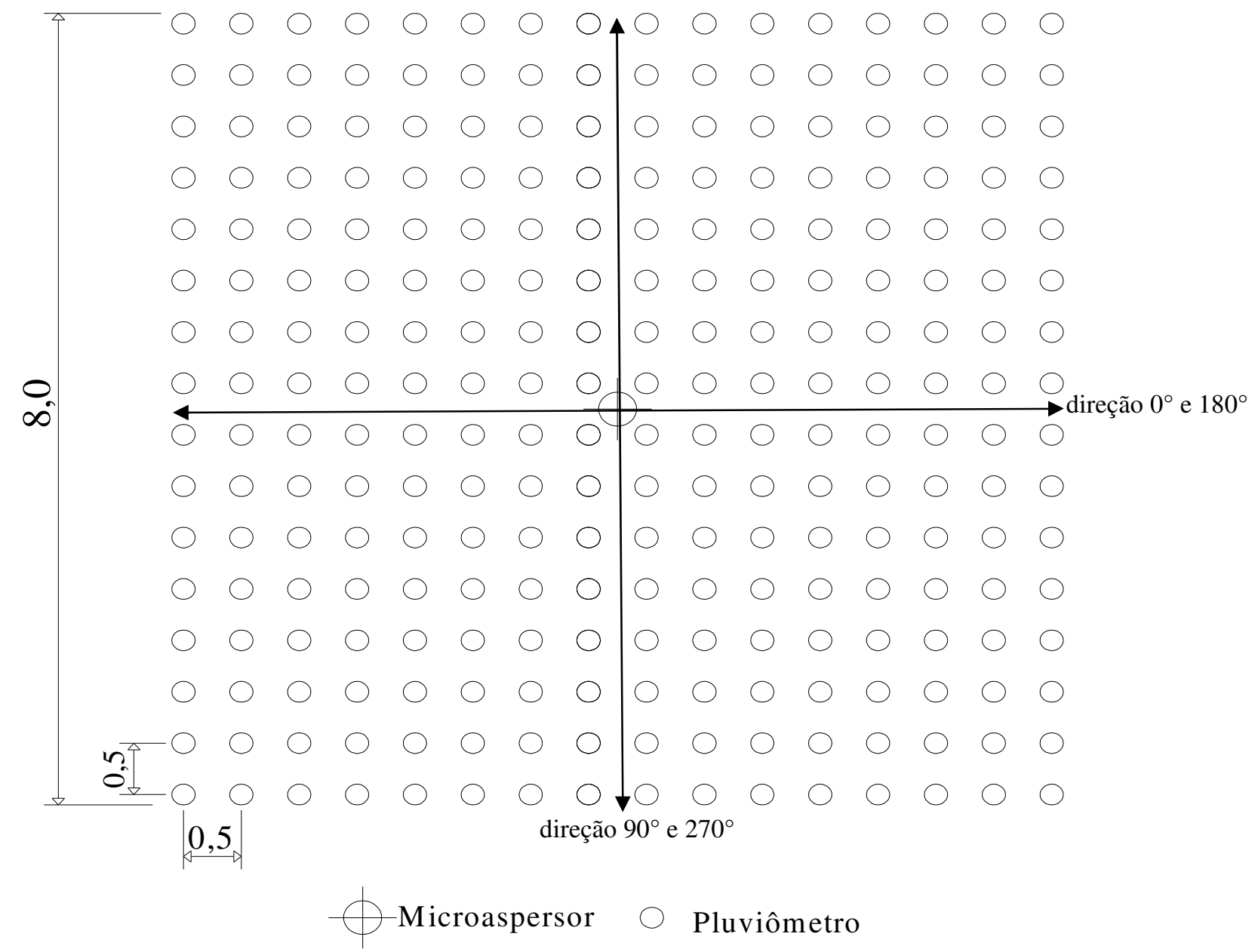

FIGURA 1. Distribuição de coletores para ensaio da uniformidade de distribuição de água. Collectors distribution to test the water distribution uniformity.

O coeficiente de uniformidade de CHRISTIANSEN (1942) foi obtido pela eq.(2), e o coeficiente de uniformidade de distribuição, pela eq.(3):

$$
\mathrm{CUC}=100\left(1-\frac{\sum|\mathrm{Xi}-\overline{\mathrm{X}}|}{\sum \mathrm{n} \overline{\mathrm{X}}}\right)
$$

em que,

CUC - coeficiente de uniformidade de Christiansen, \%;

$\mathrm{Xi}$ - quantidade medida em cada coletor, $\mathrm{mm}$;

$\overline{\mathrm{X}}=(\mathrm{X} \mathrm{i} / \mathrm{n})-$ quantidade média dos coletores, $\mathrm{mm}$, e

$\mathrm{n}$ - número de coletores. 


$$
\mathrm{CUD}=\frac{\mathrm{q}^{25}}{\mathrm{q}} 100
$$

em que,

CUD - coeficiente de uniformidade de distribuição, \%;

$\mathrm{q}^{25}$ - vazão média dos $25 \%$ menores valores de vazão, $\mathrm{L} \mathrm{h}^{-1}$, e

$\overline{\mathrm{q}}$ - média de todas as vazões observadas, $\mathrm{L} \mathrm{h}^{-1}$.

O coeficiente de uniformidade de distribuição - CUD, e o coeficiente de uniformidade de Christiansen - CUC, foram analisados considerando lâmina d'água obtida pela média das três repetições para cada pressão analisada. A simulação da uniformidade de distribuição de água, para diferentes graus de sobreposição, foi obtida para os espaçamentos entre laterais de 3,0; 4,0; 5,0 e $6,0 \mathrm{~m}$ e entre microaspersores de 1,$0 ; 1,5 ; 2,0 ; 2,5$ e $3,0 \mathrm{~m}$, utilizando-se do Software Catch $3 \mathrm{~d}$ (ALLEN, 1992).

O coeficiente de uniformidade, de acordo com a norma ABNT, NBR 14244 (1998), pode ser classificado em: excelente $>90 \%$; bom, entre 85 e $89 \%$; regular, entre 80 e $84 \%$, e ruim $<80 \%$.

\section{RESULTADOS E DISCUSSÃO}

O microaspersor novo do grupo modular apresentou CVf entre 0,89\% (150 kPa) e 2,36\% (350 kPa), e valor médio de 1,67\%, sendo classificado como bom de acordo com a ABNT (1986) e excelente segundo ASAE (1994) e Categoria A, pela ISO (1991) para todas as pressões. Para o microaspersor usado na pressão de $200 \mathrm{kPa}$, a classificação do CVq foi marginal pela ASAE (1992) e Categoria B pela ISO (1991) (Tabela 1).

Para o microaspersor novo, as vazões médias obtidas no ensaio para as pressões de 150; 200; 250; 300 e $350 \mathrm{kPa}$ foram de 96,75; 113,65; 122,53; 131,23 e 141,43 $\mathrm{L} \mathrm{h}^{-1}$, enquanto no catálogo do fabricante eram de 104; 120; 134; 147 e $159 \mathrm{~L} \mathrm{~h}^{-1}$, respectivamente, ou seja, aproximadamente $8,2 \%$ menores.

Variações menores que as obtidas por SIQUEIRA \& BARROS JÚNIOR (2003) para o microaspersor Carborundum MS V, submetido a diferentes pressões de serviço, onde as vazões variaram entre 8,57 a 15,00\% em comparação com as informações catalogadas pelo fabricante.

A equação vazão x pressão obtida foi: $\mathrm{q}=11,5336 \mathrm{H}^{0,4278}$, com coeficiente de determinação $\left(\mathrm{R}^{2}\right)$ de 0,996 , considerado um ótimo ajuste da equação. O expoente "x" da equação característica foi próximo de 0,5, que segundo KELLER \& KARMELLI (1974), caracteriza o emissor como de fluxo turbulento.

O microaspersor usado do grupo modular apresentou CVq entre 1,34\% (250 kPa) e 8,81\% (200 kPa) e valor médio de 3,33\% (Tabela 1). As vazões médias obtidas no ensaio para as pressões de 200; 250 e $300 \mathrm{kPa}$ foram de 108,56; 121,72 e 133,82 $\mathrm{L} \mathrm{h}^{-1}$, enquanto no catálogo do fabricante são de 120; 134 e $147 \mathrm{~L} \mathrm{~h}^{-1}$, respectivamente, aproximadamente 9,3\% menor.

A equação vazão x pressão obtida foi: $\mathrm{q}=8,3666 \mathrm{H}^{0,486}$, com coeficiente de determinação de 0,998. VILAS BOAS et al. (2001) avaliaram o microaspersor Dan sprinkler do grupo modular, para os diâmetros de 0,$94 ; 1,16 ; 1,41 ; 1,92$ e 2,34 para as pressões de $100 ; 150 ; 200 ; 250 ; 300$ e $350 \mathrm{kPa}$, observando que as equações características indicam que o microaspersor é tolerante à sensibilidade de variação de pressão e que a equação potencial se ajustou bem aos dados, com valores de coeficiente de determinação acima de 0,99 , semelhantes aos obtidos neste trabalho. 
TABELA 1. Valores de vazão média, desvio-padrão, coeficiente de variação de fabricação e classificação segundo ABNT (1998), ABNT (1986) e ISO (1991), do microaspersor modular novo e usado, para diferentes pressões. Average discharge rate, deviationstandard and manufacturing coefficient of variation of microsprinkler and classification according to ASAE (1994), ABNT (1986) and ISO (1991), to new and used modulate microsprinkler for different pressures.

\begin{tabular}{|c|c|c|c|c|c|c|c|c|c|c|c|c|}
\hline \multirow{3}{*}{$\begin{array}{c}\text { Pressão } \\
(\mathrm{kPa})\end{array}$} & \multirow{2}{*}{\multicolumn{2}{|c|}{$\begin{array}{l}\text { Vazão } \\
\left(\mathrm{L} \mathrm{h}^{-1}\right) \\
\end{array}$}} & \multirow{2}{*}{\multicolumn{2}{|c|}{$\begin{array}{c}\text { Desvpad } \\
\left(\mathrm{L} \mathrm{h}^{-1}\right) \\
\end{array}$}} & \multirow{2}{*}{\multicolumn{2}{|c|}{$\begin{array}{c}\text { CVf CVq } \\
(\%)\end{array}$}} & \multicolumn{6}{|c|}{ Classificação } \\
\hline & & & & & & & \multicolumn{2}{|c|}{ ASAE (1994) } & \multicolumn{2}{|c|}{ ABNT (1986) } & \multicolumn{2}{|c|}{ ISO (1991) } \\
\hline & novo & usado & novo & usado & novo & usado & novo & usado & novo & usado & novo & usado \\
\hline 150 & 96,75 & 96,95 & 0,86 & 1,76 & 0,89 & 1,82 & Exc. & Exc. & Boa & Boa & A & A \\
\hline 200 & 113,65 & 108,56 & 2,09 & 9,56 & 1,84 & 8,81 & Exc. & Mar. & Boa & Boa & A & B \\
\hline 250 & 122,53 & 121,72 & 1,61 & 1,63 & 1,31 & 1,34 & Exc. & Exc. & Boa & Boa & A & A \\
\hline 300 & 131,23 & 133,82 & 2,62 & 1,97 & 1,94 & 1,47 & Exc. & Exc. & Boa & Boa & A & A \\
\hline 350 & 141,43 & 144,98 & 3,47 & 4,64 & 2,36 & 3,20 & Exc. & Exc. & Boa & Boa & A & $\mathrm{A}$ \\
\hline
\end{tabular}

$\begin{array}{lllllll}\text { Média } & - & - & 2,13 & 3,91 & 1,67 & 3,33\end{array}$

Desvpad - desvio-padrão; A - categoria A; B - categoria B; Exc.: Excelente; Mar.: marginal

O maior CUC sem sobreposição foi de 49,5\% (200 kPa) e CUD de 49,0\% (250 kPa) para o microaspersor modular novo, e para o usado, o CUC foi de 42,9\% e o CUD de 39,9\% (150 kPa) (Tabela 2). Observa-se que o índice de CUC dos microaspersores usados foi $6,6 \%$ menor se comparado ao obtido em microaspersores novos. Essa redução pode ser atribuída a não estabilidade de rotação da asa giratória devido ao desgaste pelo tempo de uso e incrustações de impurezas nas mesmas, intensificado pela aplicação de nutrientes via água de irrigação. Valores inferiores foram obtidos por ROCHA et al. (2001) para três modelos do miniaspersor Mamkad autocompensante, onde a vazão dos emissores aumentou em até 33,2\%, quando submetidos a acréscimos de pressão, dentro do intervalo recomendado pelo fabricante.

TABELA 2. Coeficiente de uniformidade de Christiansen - CUC, e coeficiente de uniformidade de distribuição - CUD, obtidos sem sobreposição para os microaspersores do grupo modular novos e usados, para diferentes pressões. Christiansen's uniformity coefficient - CUC and distribution - CUD obtained without overlap for new and used microsprinkler of modulate group for different pressures.

\begin{tabular}{cccccc}
\hline \multirow{2}{*}{ Pressão $(\mathrm{kPa})$} & \multicolumn{2}{c}{ CUC $(\%)$} & & \multicolumn{2}{c}{ CUD (\%) } \\
\cline { 2 - 3 } \cline { 5 - 5 } & Novos & Usados & & Novos & Usados \\
\hline 150 & 39,7 & 42,9 & & 38,4 & 39,9 \\
200 & 49,5 & 41,9 & & 42,0 & 38,0 \\
250 & 49,2 & 38,4 & & 49,0 & 38,3 \\
300 & 45,0 & 38,5 & & 42,1 & 38,0 \\
350 & 39,0 & 36,4 & & 41,1 & 37,9 \\
\hline
\end{tabular}

Considerando diferentes graus de sobreposições, verificou-se que a maior uniformidade ocorreu no espaçamento de 3,0 x 1,0 m (95,5\%), na pressão de $350 \mathrm{kPa}$, para o microaspersor novo (Tabela 3), e no espaçamento de 4,0 x 2,0 m (97,3\%), na pressão de $250 \mathrm{kPa}$, para o microaspersor usado (Tabela 4). Ocorreu redução do CUC com aumento do espaçamento entre os microaspersores, embora houvesse espaçamentos entre laterais de até $5,0 \mathrm{~m}$ em todas as pressões para modelos novos e usados. Exceção ocorreu para os espaçamentos 4,0 x 2,5 m; 4,0 × 3,0 m; 5,0 x 2,5 m e 5,0 x 3,0 m na pressão de $150 \mathrm{kPa}$, para o microaspersor novo, apresentarando CUC superior a $80 \%$, sendo este valor mínimo recomendável para um sistema de irrigação pressurizado, segundo GOMES (1999). 
TABELA 3. Coeficientes de uniformidade de Christiansen - CUC, e de distribuição - CUD, do microaspersor do grupo modular novo, bocal laranja, para diferentes pressões e grau de sobreposição. Christiansen's uniformity coefficient - CUC and distribution CUD of new microsprinkler of modulate group, orange nozzle for different pressures and overlap.

\begin{tabular}{|c|c|c|c|c|c|c|c|c|}
\hline \multirow{4}{*}{$\begin{array}{l}\text { Espaçamento } \\
\text { entre } \\
\text { microaspersores } \\
\text { (m) }\end{array}$} & \multicolumn{4}{|c|}{ CUC (\%) } & \multicolumn{4}{|c|}{ CUD $(\%)$} \\
\hline & \multicolumn{8}{|c|}{ Espaçamento entre Laterais $(\mathrm{m})$} \\
\hline & 3,0 & 4,0 & 5,0 & 6,0 & 3,0 & 4,0 & 5,0 & 6,0 \\
\hline & \multicolumn{8}{|c|}{$150 \mathrm{kPa}$} \\
\hline 1,0 & 91,6 & 86,0 & 86,7 & 78,9 & 92,6 & 86,9 & 89,2 & 97,9 \\
\hline 1,5 & 90,4 & 85,1 & 86,0 & 77,3 & 91,4 & 88,7 & 88,2 & 77,5 \\
\hline 2,0 & 90,0 & 84,3 & 84,9 & 76,5 & 91,4 & 86,6 & 87,4 & 79,0 \\
\hline 2,5 & 85,5 & 78,6 & 76,1 & 70,9 & 86,4 & 76,2 & 80,1 & 76,4 \\
\hline \multirow[t]{2}{*}{3,0} & 82,6 & 73,8 & 76,6 & 72,1 & 82,2 & 76,7 & 82,0 & 72,8 \\
\hline & \multicolumn{8}{|c|}{$200 \mathrm{kPa}$} \\
\hline 1,0 & 93,4 & 92,0 & 89,0 & 78,5 & 94,5 & 91,9 & 89,2 & 80,6 \\
\hline 1,5 & 93,3 & 91,3 & 88,6 & 78,3 & 94,2 & 92,8 & 89,8 & 80,3 \\
\hline 2,0 & 92,5 & 90,3 & 87,7 & 77,7 & 93,5 & 90,3 & 88,1 & 78,9 \\
\hline 2,5 & 88,5 & 87,8 & 82,9 & 75,1 & 88,1 & 85,8 & 84,5 & 79,1 \\
\hline \multirow[t]{2}{*}{3,0} & 89,3 & 81,6 & 80,2 & 76,6 & 89,9 & 81,6 & 83,9 & 77,7 \\
\hline & \multicolumn{8}{|c|}{$250 \mathrm{kPa}$} \\
\hline 1,0 & 94,3 & 93,0 & 88,9 & 76,8 & 95,3 & 94,0 & 88,9 & 78,8 \\
\hline 1,5 & 92,7 & 92,2 & 88,1 & 76,5 & 94,8 & 93,5 & 89,5 & 78,3 \\
\hline 2,0 & 92,9 & 91,4 & 87,0 & 75,0 & 92,9 & 91,1 & 87,9 & 78,0 \\
\hline 2,5 & 89,4 & 89,4 & 84,5 & 74,0 & 88,8 & 88,3 & 85,9 & 78,6 \\
\hline \multirow[t]{2}{*}{3,0} & 91,0 & 84,1 & 81,9 & 75,7 & 90,8 & 84,4 & 83,9 & 76,5 \\
\hline & \multicolumn{8}{|c|}{$300 \mathrm{kPa}$} \\
\hline 1,0 & 95,0 & 95,1 & 86,7 & 71,9 & 96,0 & 95,2 & 88,3 & 74,4 \\
\hline 1,5 & 93,8 & 93,5 & 85,6 & 71,8 & 95,0 & 94,6 & 87,5 & 73,1 \\
\hline 2,0 & 93,5 & 92,6 & 85,4 & 70,7 & 92,8 & 91,8 & 86,6 & 73,6 \\
\hline 2,5 & 91,8 & 91,4 & 84,0 & 70,4 & 91,5 & 91,0 & 86,9 & 74,2 \\
\hline \multirow[t]{2}{*}{3,0} & 92,0 & 86,9 & 82,6 & 71,4 & 92,2 & 86,8 & 83,9 & 72,5 \\
\hline & \multicolumn{8}{|c|}{$350 \mathrm{kPa}$} \\
\hline 1,0 & 95,5 & 95,0 & 83,5 & 67,7 & 96,2 & 95,4 & 86,8 & 72,0 \\
\hline 1,5 & 94,6 & 94,4 & 83,7 & 67,7 & 95,3 & 94,6 & 85,9 & 71,0 \\
\hline 2,0 & 93,4 & 93,0 & 82,9 & 67,3 & 93,3 & 93,8 & 85,0 & 71,8 \\
\hline 2,5 & 92,4 & 91,7 & 82,0 & 66,7 & 92,1 & 91,6 & 85,3 & 71,7 \\
\hline 3,0 & 93,2 & 89,3 & 81,9 & 67,4 & 92,9 & 88,4 & 83,1 & 70,2 \\
\hline
\end{tabular}


TABELA 4. Coeficientes de uniformidade de Christiansen - CUC, e de distribuição - CUD, do microaspersor do grupo modular usado, bocal laranja, para diferentes pressões e grau de sobreposição. Christiansen's uniformity coefficient - CUC and distribution CUD of used microsprinkler of modulate group, orange nozzle for different pressures and overlap.

\begin{tabular}{|c|c|c|c|c|c|c|c|c|}
\hline \multirow{4}{*}{$\begin{array}{c}\text { Espaçamento } \\
\text { entre } \\
\text { microaspersores } \\
(\mathrm{m}) \\
\end{array}$} & \multicolumn{4}{|c|}{ CUC (\%) } & \multicolumn{4}{|c|}{ CUD $(\%)$} \\
\hline & \multicolumn{8}{|c|}{ Espaçamento entre laterais (m) } \\
\hline & 3,0 & 4,0 & 5,0 & 6,0 & 3,0 & 4,0 & 5,0 & 6,0 \\
\hline & \multicolumn{8}{|c|}{$150 \mathrm{kPa}$} \\
\hline 1,0 & 91,4 & 93,5 & 88,5 & 78,4 & 93,1 & 94,2 & 89,3 & 82,1 \\
\hline 1,5 & 91,0 & 91,8 & 87,6 & 77,8 & 93,0 & 91,0 & 88,8 & 81,8 \\
\hline 2,0 & 89,5 & 91,1 & 85,3 & 77,0 & 89,6 & 91,8 & 87,4 & 81,0 \\
\hline 2,5 & 85,6 & 85,1 & 81,5 & 73,7 & 87,4 & 83,4 & 83,2 & 80,0 \\
\hline \multirow[t]{2}{*}{3,0} & 88,4 & 83,8 & 82,5 & 75,9 & 89,9 & 84,6 & 85,5 & 78,7 \\
\hline & \multicolumn{8}{|c|}{$200 \mathrm{kPa}$} \\
\hline 1,0 & 93,7 & 96,4 & 86,3 & 72,3 & 93,1 & 96,3 & 89,0 & 77,7 \\
\hline 1,5 & 93,8 & 95,2 & 85,6 & 72,2 & 94,9 & 95,0 & 88,9 & 76,7 \\
\hline 2,0 & 92,2 & 94,1 & 84,8 & 71,9 & 92,6 & 94,2 & 87,3 & 76,4 \\
\hline 2,5 & 87,8 & 89,7 & 82,0 & 69,8 & 88,8 & 88,5 & 83,8 & 76,5 \\
\hline \multirow[t]{2}{*}{3,0} & 91,9 & 87,3 & 82,5 & 71,5 & 93,1 & 88,3 & 84,9 & 75,7 \\
\hline & \multicolumn{8}{|c|}{$250 \mathrm{kPa}$} \\
\hline 1,0 & 95,2 & 97,3 & 85,2 & 69,9 & 95,9 & 96,9 & 88,7 & 75,7 \\
\hline 1,5 & 95,2 & 96,0 & 85,0 & 69,9 & 95,8 & 96,3 & 88,0 & 75,1 \\
\hline 2,0 & 92,8 & 93,7 & 84,1 & 69,5 & 93,8 & 94,3 & 86,5 & 75,0 \\
\hline 2,5 & 88,7 & 90,4 & 82,1 & 68,0 & 89,7 & 90,2 & 84,6 & 74,3 \\
\hline \multirow[t]{2}{*}{3,0} & 91,9 & 89,6 & 82,3 & 68,9 & 92,7 & 90,1 & 84,3 & 74,2 \\
\hline & \multicolumn{8}{|c|}{$300 \mathrm{kPa}$} \\
\hline 1,0 & 94,4 & 96,2 & 83,7 & 68,8 & 95,5 & 96,6 & 89,3 & 74,2 \\
\hline 1,5 & 93,9 & 94,1 & 83,4 & 68,2 & 94,7 & 95,0 & 87,4 & 73,5 \\
\hline 2,0 & 92,6 & 92,5 & 83,2 & 68,7 & 93,1 & 92,9 & 87,4 & 73,2 \\
\hline 2,5 & 87,8 & 90,2 & 81,7 & 67,1 & 89,6 & 89,9 & 84,1 & 72,8 \\
\hline \multirow[t]{2}{*}{3,0} & 91,5 & 90,6 & 82,1 & 67,7 & 93,1 & 91,1 & 84,4 & 72,8 \\
\hline & \multicolumn{8}{|c|}{$350 \mathrm{kPa}$} \\
\hline 1,0 & 95,7 & 96,2 & 82,9 & 67,1 & 96,3 & 96,4 & 88,9 & 72,4 \\
\hline 1,5 & 94,9 & 94,3 & 82,5 & 66,8 & 95,5 & 95,3 & 87,0 & 72,1 \\
\hline 2,0 & 92,5 & 91,5 & 82,3 & 66,8 & 93,5 & 91,6 & 86,3 & 71,7 \\
\hline 2,5 & 88,3 & 90,1 & 81,0 & 65,5 & 90,0 & 91,1 & 84,2 & 71,4 \\
\hline 3,0 & 92,1 & 91,4 & 81,5 & 66,0 & 93,2 & 91,6 & 83,9 & 71,0 \\
\hline
\end{tabular}

Nas Figuras 2a, 2b, 2c e 2d, apresentam-se as curvas de distribuição de água sem sobreposição dos microaspersores modular (novos e usados), onde se observa maior precipitação próxima ao microaspersor (próximo a $7 \mathrm{~mm} \mathrm{~h}^{-1}$ para modelo novo e $10 \mathrm{~mm} \mathrm{~h}^{-1}$ para usado) reduzindo desta forma a precipitação ao longo dos espaçamentos, embora exista "redução" na região imediatamente próxima do emissor, influenciada pela "ponte" do microaspersor. 

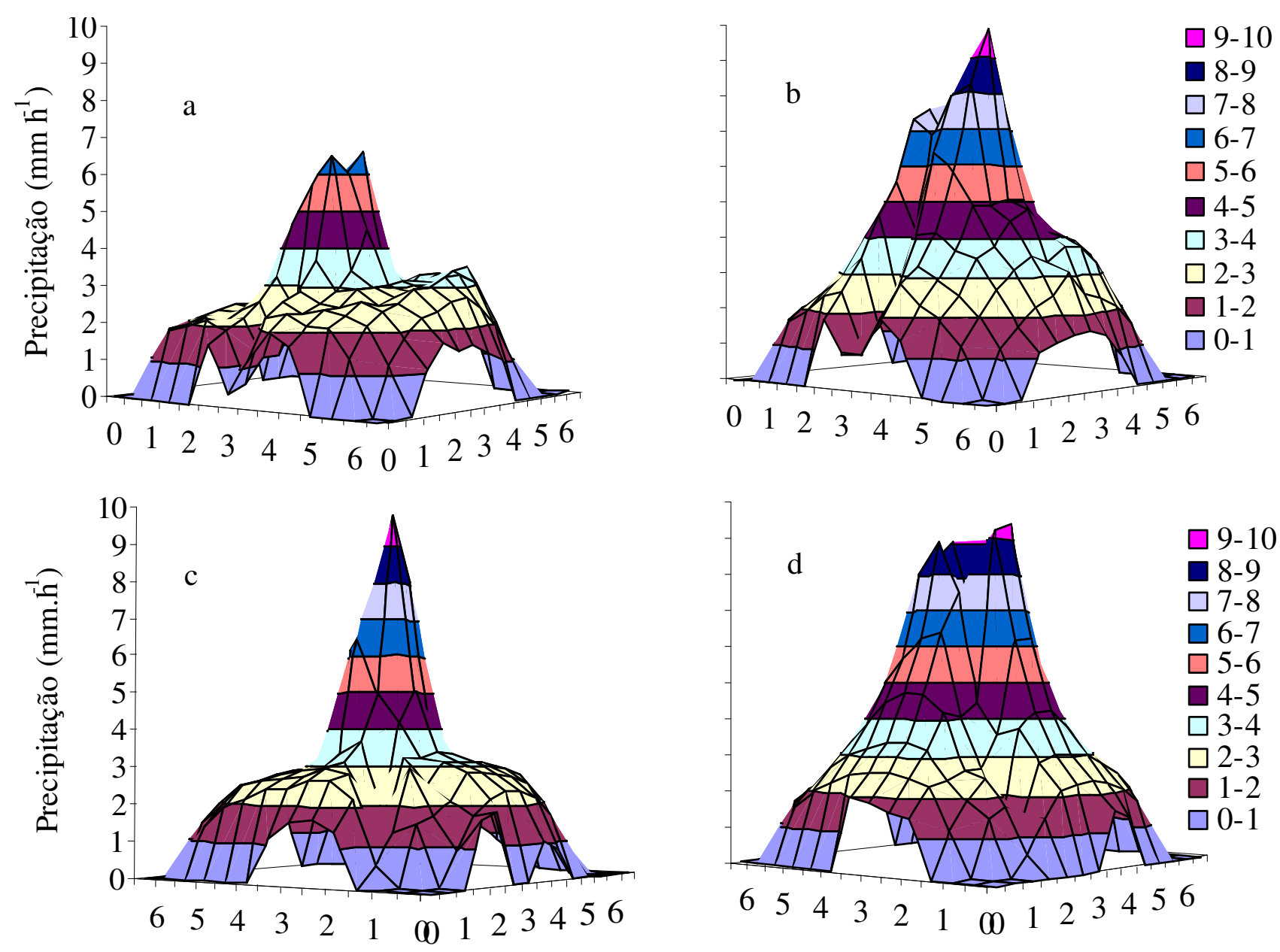

FIGURA 2. Curvas de mesma precipitação (3D) para o microaspersor do grupo modular novo, bocal laranja, para a maior (1a), (200 kPa) e menor uniformidade (1b), (350 kPa) e para o microaspersor usado, para a maior $(1 \mathrm{c}),(150 \mathrm{kPa})$ e menor uniformidade (1d), $(350 \mathrm{kPa})$, sem sobreposição. Curves of the same precipitation (3D) to the new microsprinkler of modulate group, orange nozzle for the higher (1a), (200 kPa) and the lower uniformity $(1 \mathrm{~b}),(350 \mathrm{kPa})$ and for the used microsprinkler for higher ( 1c), (150 kPa) and lower uniformity (1d), (350 kPa), without overlap.

As curvas de precipitação para os dois diâmetros, ou seja, na direção de $0^{\circ}$ e $180^{\circ}$ e na direção de $90^{\circ}$ e $270^{\circ}$, apresentaram maior precipitação a uma distância de $1,0 \mathrm{~m}$ do microaspersor para o modelo novo, enquanto o modelo usado apresentou uma redução no valor da precipitação a uma distância de $0,5 \mathrm{~m}$ e logo após uma elevação, reduzindo seu valor posteriormente, para os modelos novos e usados (Figuras 3a, 3b, 3c e 3d). Resultados semelhantes foram obtidos por TAGLIAFERRE et al. (2003) para o microaspersor MF, da marca Carborundum, onde obtiveram perfil de precipitação com altas lâminas próximo ao microaspersor, principalmente nas pressões de 160 e $200 \mathrm{kPa}$.

O raio de alcance e os pontos de maior precipitação são dependentes das condições de funcionamento de cada marca e modelo de equipamento, como pode ser observado no trabalho realizado por SILVA \& SILVA (2003), que obtiveram para o microdifusor autocompensante DAN JET 7200, nas pressões de 49; 98; 147; 196; 245; 294 e $343 \mathrm{kPa}$, maiores valores de precipitação entre 1,20 e 2,10 m de distância, em relação à haste de sustentação, e maior concentração de jatos na faixa de 1,80 a 2,40 m. Já CONCEIÇÃ̃O \& COELHO (2004) determinaram a distribuição radial do volume de água aplicada por microaspersores DAN 2001 autocompensantes, que utilizam rotores preto (bocais violeta, cinza e marrom) e azul (bocais azul, verde, laranja e amarelo), observando que os maiores percentuais dos volumes de água aplicados ficaram entre as distâncias 
de $1,2 \mathrm{~m}$ e $1,8 \mathrm{~m}$, para os bocais violeta, cinza e marrom; entre $1,8 \mathrm{~m}$ e $2,4 \mathrm{~m}$ para o bocal azul, e entre 2,4 m e 3,0 m, para os bocais verde, laranja e amarelo.
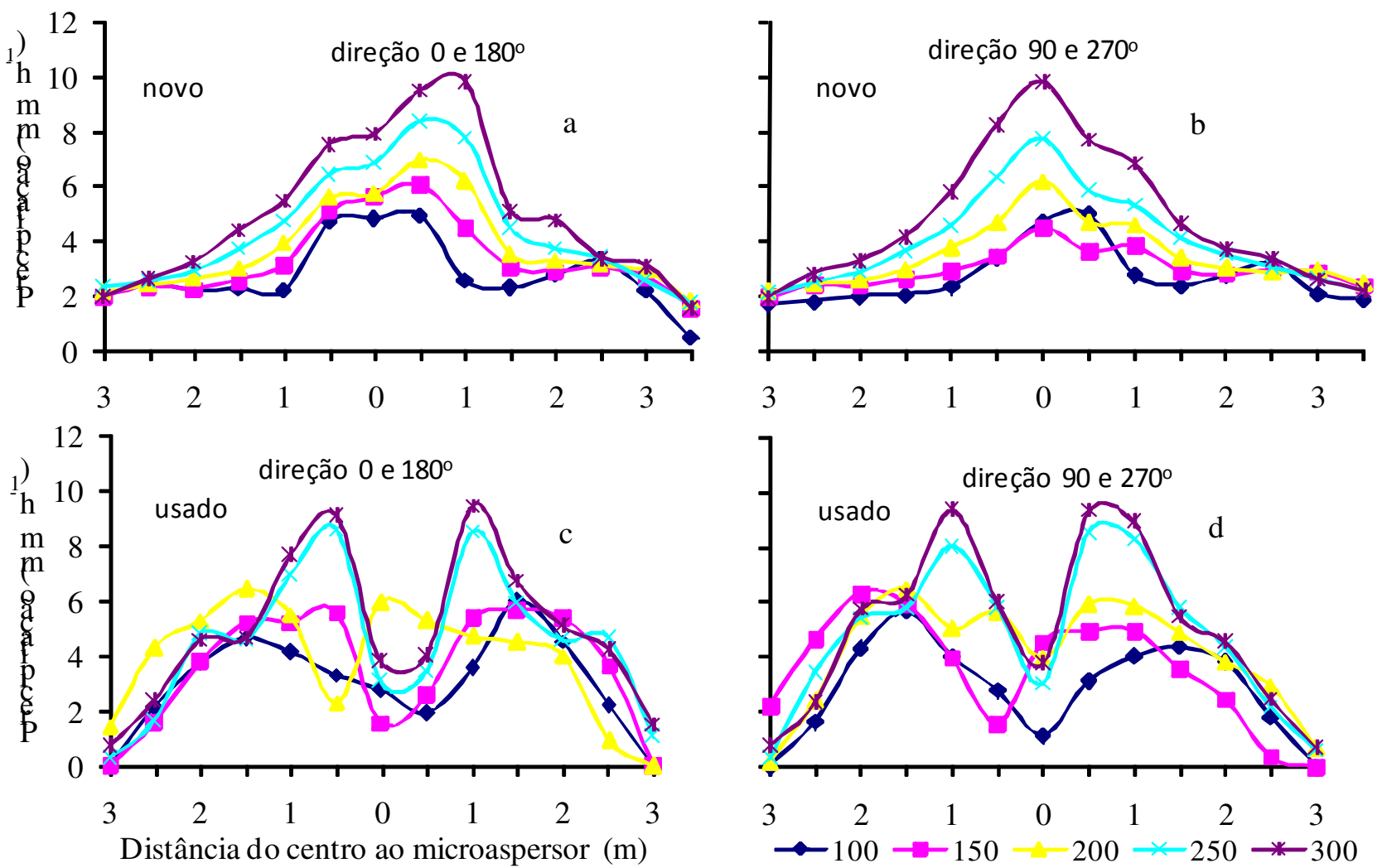

FIGURA 3. Curvas do padrão de distribuição de água para o microaspersor modular, bocal laranja, novo, para a direção de $0^{\circ}$ e $180^{\circ}$ (a), $90^{\circ}$ e $270^{\circ}$ (b) e usado na direção de $0^{\circ}$ e $180^{\circ}$ (c), $90^{\circ}$ e $270^{\circ}$ (d) para diferentes pressões. Distribution curves of the water pattern to the new modulate microsprinkler orange nozzle to the direction of $0^{\circ}$ and $180^{\circ}$ (a), $90^{\circ}$ and $270^{\circ}$ (b) and used in the direction of $0^{\circ}$ and $180^{\circ}$ (c) $90^{\circ}$ and $270 \circ$ (d) for different pressures.

O microaspersor de bocal laranja, usado, reduziu o raio de alcance de $0,5 \mathrm{~m}$ em relação ao de bocal novo para todas as pressões (Figuras 3a, 3b, 3c e 3d). Esse comportamento, no entanto, nunca é levado em consideração no momento da escolha de um microaspersor, podendo comprometer o grau de sobreposição do jato de água, e consequentemente, os índices de uniformidade ao longo do tempo de uso. Essa redução do alcance do jato de água está relacionada, no caso específico, ao ressecamento da haste giratória (bailarina) e aderência de impurezas, especialmente sais, bem como desgaste dos contatos e componentes giratórios, alterando, possivelmente, o ângulo do jato de água.

\section{CONCLUSÕES}

O microaspersor do grupo modular novo e usado apresentaram excelentes valores de coeficiente de variação de fabricação.

O aumento da distância entre laterais e microaspersores, em todas as pressões para os modelos novos e usados, reduziu os índices de CUC e CUD. O espaçamento entre laterais de 6,0 m, em todos os espaçamentos entre microaspersores e pressões avaliadas, não é indicado, por apresentar valores de CUC inferior a $80 \%$. fabricante.

O microaspersor, novo e usado, apresentou vazões inferiores às indicadas pelo catálogo do

O alcance do jato de água do microaspersor usado foi menor em relação ao bocal novo. 


\section{REFERÊNCIAS}

ABNT. ASSOCIAÇÃO BRASILEIRA DE NORMAS TÉCNICAS. Requisitos mínimos para elaboração de projeto de sistema de irrigação localizada. PNBR 12-02-08 - 022. São Paulo, 1986. $8 \mathrm{p}$.

ABNT. ASSOCIAÇÃO BRASILEIRA DE NORMAS TÉCNICAS. Equipamentos de irrigação mecanizada - Pivô central, lateral móvel providos de emissores fixos ou rotativos - Determinação da uniformidade de distribuição de água. NBR 14244. São Paulo, (1998).

ALLEN, R.G. Sprinkler pattern analysis: user's manual. $3^{\text {rd }}$ ed. Logan Utah. Department of Biological and Irrigation Engineering, Utah State University, Logan. 1992. 14 p.

ASAE. Equipamentos de irrigação mecanizada - Pivô central, lateral móvel providos de emissores fixos ou rotativos - Determinação da uniformidade de distribuição de água. ABNT, NBR 14244. São Paulo, 1992.

ASAE. AMERICAN SOCIETY OF AGRICULTURAL ENGINEERS. Design and installation of microirrigation system. St. Joseph: ASAE EP405.1, 1994. p.724-727.

ASSAD, E.D. Chuva nos cerrados: análise e espacialização. Brasília: Embrapa/SPI, 1994. 423 p.

BRASIL. Ministério do Meio Ambiente. GEO Brasil: Recursos Hídricos: componentes da série de relatórios sobre o estado e perspectivas do meio ambiente no Brasil. Brasília: MMA/ANA, 2007. $264 \mathrm{p}$.

CHRISTIANSEN, J.E. Irrigation by sprinkling. Berkeley: University of Califórnia, 1942.124 p.

CONCEIÇÃO, M.A.F.; COELHO, R.D. Distribuição do volume de água aplicado pelo microaspersor DAN 2001. Irriga, Botucatu, v.9, n.3, p.289-295, 2004.

GOMES, H.P. Hidráulica dos sistemas pressurizados: aspersão e gotejamento. 3.ed. Campina Grande: Universidade Federal da Paraíba, 1999. 412 p.

ISO. INTERNATIONAL ORGANIZATION FOR STANDARDIZATION. ISO 9260/1.

Agricultural irrigation equipment - emitters - specification and test methods, Switzerland. 1991. $6 \mathrm{p}$.

KELLER, J.; BLIESNER, R.D. Sprinkle and trickle irrigation. New York: Van Nostrand Reinhold, 1990. p.42-163.

KELLER, J.; KAMERLI, D. Evaluation of a trickle irrigation system, In: INTERNATIONAL DRIP IRRIGATION CONGRESS, 2., 1974, San Diego. Anais... p.287-292.

MAZZER, H.R.; VILAS BOAS, M.A.; SAMPAIO, S.C. Comparação de dois métodos de ensaios radiais para microaspersores. In: CONGRESSO BRASILEIRO DE ENGENHARIA AGRÍCOLA, 32., 2003, Goiânia. Anais... Goiânia: Associação Brasileira de Engenharia Agrícola, 2003. 1 CD-ROM.

QUEIROZ, S.O.P. Métodos para avaliação de salinização do solo sob condições de ambiente protegido. 2004. $101 \mathrm{f}$. Tese (Doutorado em Engenharia Agrícola) - Universidade Estadual de Campinas, Campinas, 2004.

ROCHA, F.A.; CÉSAR, J.H.F.; MELLO, C.R.; ROCHA, F.S.; PEREIRA, G.M. Caracterização hidráulica de três modelos do miniaspersor Mamkad autocompensante. Revista Brasileira de Engenharia Agrícola e Ambiental, Campina Grande, v.5, n.1, p.6-9, 2001.

SANTIAGO, F.S.; MONTENEGRO, A.A.A.; MONTENEGRO, S.M.G.L. Avaliação de parâmetros hidráulicos e manejo da irrigação por microaspersão em áreas de assentamento. Engenharia Agrícola, Jaboticabal, v.24, n.3, p.632-643, 2004.

SAPIR, E.; SNEH, N. Riego por aspersion. Tel Aviv: MASHAV-CINADCO, 2002. 127 p. 
SCALOPPI, J.E.; DIAS, K.F.S. Relação entre a pressão de operação e a uniformidade de distribuição de água de aspersores rotativos por impacto. In: CONGRESSO BRASILEIRO DE ENGENHARIA AGRÍCOLA, 26., 1996, Bauru. Anais... Bauru: Associação Brasileira de Engenharia Agrícola, 1996. 1 CD-ROM.

SILVA, R.A.; SILVA, A.M. Avaliação das características hidráulicas do microdifusor autocompesante DAN JET 7200. Ciência Agrotécnica, Lavras, v.27, n.4, p.873-878, 2003.

SIQUEIRA, E.C.; BARROS JÚNIOR, G. Determinação do coeficiente de variação e vazão do microaspersor Carburundum MS V versus pressão. In: CONGRESSO BRASILEIRO DE ENGENHARIA AGRÍCOLA, 32, 2003, Goiânia. Anais... Goiânia: Associação Brasileira de Engenharia Agrícola, 2003. 1 CD-ROM.

SOUZA, J.A.R.; DENÍCULLI, W.; BATISTA, R.O.; VAL, J.C.C.; MATOS, A.T. Caracterização hidráulica de microaspersor aplicando água limpa, água residuária de avicultura e de bovinocultura. Engenharia na Agricultura, Viçosa-MG, v.13, n.3, p.161-172, 2005.

TAGLIAFERRE, C.; OLIVEIRA, R.A.; DENÍCULLI, W.; ROBERTO CECON, P.; MARTINEZ, M.A. Características hidráulicas do microaspersor MF. In: CONGRESSO BRASILEIRO DE ENGENHARIA AGRÍCOLA, 32., 2003, Goiânia. Anais... Goiânia: Associação Brasileira de Engenharia Agrícola, 2003. 1 CD-ROM.

VILAS BOAS, M.A.; MACEDO JUNIOR, E.K.; SAMPAIO, S.C.; VALIATI, M.I. Caracterização hidráulica do microaspersor Dan sprinkler grupo modular. Irriga, Botucatu, v.6, n.1, p.29-36, 2001. 